Pacific Journal of Mathematics

AUTOMORPHISMS AND EQUIVALENCE IN YON NEWMAN 


\title{
AUTOMORPHISMS AND EQUIVALENCE IN VON NEUMANN ALGEBRAS
}

\section{ERLING STøRMER}

\begin{abstract}
Let $\Re$ be a von Neumann algebra acting on a Hilbert space $\mathscr{S}_{\text {. }}$ Let $G$ be a group and let $t \rightarrow U_{t}$ be a unitary representation of $G$ on $\mathfrak{S}$ such that $U_{t}^{*} \Re U_{t}=\Re$ for all $t \in G$. Two projections $E$ and $F$ in $\Re$ are called $G$-equivalent, written $E \sim \sim_{G} F$, if there is for each $t \in G$ an operator $T_{t} \in \Re$ such that $E=\sum_{t \in G} T_{t} T_{t}^{*}, F=\sum_{t \in G} U_{t}^{*} T_{t}^{*} T_{t} U_{t}$. The main results in this paper state that this relation is indeed an equivalence relation (Thm. 1), that "semi-finiteness" is equivalent to the existence of a faithful normal semi-finite $G$-invariant trace on $\Re^{+}$(Thm. 2), and that "finiteness" together with countable decomposability of $\Re$ is equivalent to the existence of a faithful normal finite $G$-invariant trace on $\Re$ (Thm. 3 ).
\end{abstract}

There are two approaches which can be used to prove these theorems. The most natural one would be to develop a comparison theory for projections in $\Re$ and then to construct the traces. This can be done by means of modifications and extensions of the theory developed by Kadison and Pedersen [4]. The other approach, which we shall follow, is to consider the cross product $\Re \times G$, and then show that the canonical imbedding of $\mathfrak{R}$ into the von Neumann algebra $\Re \times G$ is close to being an isomorphism of $\Re$ with the structure of $G$-equivalence into $\Re \times G$ with the usual equivalence relation between projections.

Our main theorems form a link between von Neumann algebras and ergodic theory. If $G$ is the one element group the equivalence relation $\sim_{G}$ reduces to the usual one defined by Murray and von Neumann [8] for projections in a von Neumann algebra. We thus obtain extensions of the theorems on existence of traces in finite and semi-finite von Nemann algebras. If the von Neumann algebra $\Re$ is abelian we show (Thm. 5), using theorems on the existence of invariant measures, that the equivalence relation $\sim_{G}$ is the same as the one defined by Hopf [3] in ergodic theory. He showed that, with some extra assumptions, "finiteness" of the partial ordering is equivalent to the existence of an invariant nomal state. Later on the "semifinite" case was taken care of by Kawada [6] in a well ignored paper, and then independently by Halmos [2]. Thus our theorems are also generalizations of well known results on invariant measures.

We refer the reader to the book of Dixmier [1] for the theory of von Neumann algebras. The author is indepted to the referee for 
several valuable comments.

2. Statements of results. In the present section we state the main results and definitions. The proofs will be given in $\S 3$.

THEOREM 1. Let $\Re$ be a von Neumann algebra acting on a Hilbert space $\mathfrak{S}_{\text {. }}$ Let $G$ be a group and $t \rightarrow U_{t}$ a unitary representation of $G$ on $\mathfrak{S}$ such that $U_{t}^{*} \mathfrak{R} U_{t}=\mathfrak{R}$ for all $t \in G$. If $E$ and $F$ are projections in $\Re$ we write $E \sim_{G} F$ if for each $t \in G$ there is an operator $T_{t} \in \Re$ such that

$$
E=\sum_{t \in G} T_{t} T_{t}^{*}, \quad F=\sum_{t \in G} U_{t}^{*} T_{t}^{*} T_{t} U_{t} \cdot
$$

Then $\sim_{G}$ is an equivalence relation on the projections in $\Re$.

REMARK 1. If $G$ is the one element group then the equivalence relation $\sim_{G}$ is the same as the usual equivalence relation $\sim$ for projections in a von Neumann algebra.

REMARK 2. If $G$ is the additive group of $\Re$ and the representation $t \rightarrow U_{t}$ is the trivial representation, so $U_{t}=I$ for $t \in G$, then the equivalence relation $\sim_{G}$ is the one defined by Kadison and Pedersen [4, Def. A].

REMARK 3. If $\Re$ is abelian and countably decomposable the equivalence relation $\sim_{G}$ coincides with the one defined by Hopf [3] in ergodic theory. For this see Theorem 5 and Remark 6.

REMARK 4. If $E$ and $F$ are equivalent projections in $\Re$, i.e. there is a partial isometry $V \in \Re$ such that $E=V V^{*}, F=V^{*} V$, then $E \sim{ }_{G} F$. This is clear from the definition of $\sim_{G}$, putting $T_{e}=V, T_{t}=0$ for $t \neq e$.

DEFINITION 1. With notation as in Theorem 1 we say two projections $E$ and $F$ in $\Re$ are $G$-equivalent if $E \sim{ }_{G} F$. We write $E \prec_{G} F$ if $E \sim{ }_{G} F_{0} \leqq F$. A projection $F$ is said to be $\sim_{G}$-finite if $E \leqq F$ and $E \sim_{G} F$ implies $E=F . \quad \Re$ is said to be $\sim_{G}-$ finite if the identity operator $I$ is $\sim_{G}$-finite. $\Re$ is said to be $\sim_{G}$-semi-finite if every nonzero projection in $\Re$ majorizes a nonzero $\sim_{G}$-finite projection.

THEOREM 2. With notation as in Theorem 1 there exists a faithful normal semi-finite G-invariant trace on $\mathfrak{R}^{+}$if and only if $\mathfrak{R}$ is $\sim_{G}$-semi-finite.

THEOREM 3. With notation as in Theorem 1 there exists a faith- 
ful normal finite $G$-invariant trace on $\Re$ if and only if $\Re$ is $\sim_{G^{-}}$ finite and countably decomposable.

3. Proofs. We first introduce some notation and follow [1, Ch. $I$, §9] closely. Following the notation in Theorem $1 \Re \operatorname{acts}$ on a Hilbert space $\mathfrak{S}, G$ is a group, considered as a discrete group, and $t \rightarrow U_{t}$ is a unitary representation of $G$ on $\mathfrak{S}$ such that $U_{t}^{*} \Re U_{t}=\Re$ for all $t \in G$. For $t \in G$ let $\mathfrak{S}_{t}$ be a Hilbert space of the same dimen-

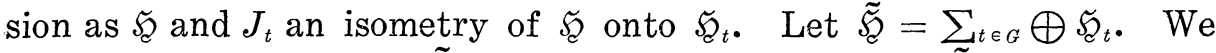
write an operator $R \in \mathfrak{B}(\tilde{\mathfrak{F}})$ - the bounded operators on $\tilde{\mathfrak{F}}$-as a matrix $\left(R_{s, t}\right)_{s, t \in \mathfrak{x}}$, where $R_{s, t}=J_{s}^{*} R J_{t} \in \mathfrak{B}(\mathfrak{S})$. For each $T \in \Re$ let $\Phi(T)$ denote the element in $\mathfrak{B}(\tilde{\mathfrak{K}})$ with matrix $\left(R_{s, t}\right)$, where $R_{s, t}=0$ if $s \neq t$, and $R_{s, s}=T$ for all $s \in G$. Then $\Phi$ is a ${ }^{*}$-isomorphism of $\Re$ onto a von Neumann subalgera $\tilde{\mathfrak{R}}$ of $\mathfrak{B}(\tilde{\mathfrak{S}})$. For $y \in G$ let $\widetilde{U}_{y}$ be the operator in $\mathfrak{B}(\tilde{\mathfrak{E}})$ with matrix $\left(R_{s, t}\right)$, where $R_{s, t}=0$ if $s t^{-1} \neq y, R_{y t, t}=U_{y}$ for all $t \in G$. Then (see [1, Ch. I, §9]) $y \rightarrow \widetilde{U}_{y}$ is a unitary representation of $G$ on $\tilde{\mathfrak{N}}$ such that

$$
\widetilde{U}_{y}^{*} \Phi(T) \widetilde{U}_{y}=\Phi\left(U_{y}^{*} T U_{y}\right), \quad y \in G, T \in \Re .
$$

If $\mathfrak{B}$ denotes the von Neumann algebra generated by $\tilde{\mathfrak{R}}$ and the $\widetilde{U}_{y}, y \in G$, then each operator in $\mathfrak{B}$ is represented by a matrix $\left(R_{s, t}\right)$ where $R_{s, t}=T_{s t-1} U_{s t^{-1}}, T_{s t^{-1}} \in \Re$.

We denote by $\Re^{c}$ the von Neumann subalgebra of $\Re$ consisting of the $G$-invariant operators in $\Re$. $\mathbb{C}$ shall denote the center of $\Re$, and $\mathfrak{D}$ shall denote $\mathfrak{C} \cap \mathfrak{R}^{a}$. Whenever we write $P \sim Q$ for two projections in $\mathfrak{B}$ we shall mean they are equivalent as operators in $\mathfrak{B}$, i.e. there is a partial isometry $V \in \mathfrak{B}$ such that $V V^{*}=P, V^{*} V=Q$, and we shall not consider $P$ and $Q$ as equivalent in a von Neumann subalgebra of $\mathfrak{B}$. The next lemma includes Theorem 1 and shows more, namely that $\sim_{G}$-equivalence is the same as equivalence in $\mathfrak{B}$.

Lemma 1. Let $E$ and $F$ be projections in $\Re$. Then $E \sim_{G} F$ if and only if $\Phi(E) \sim \Phi(F)$. Hence $\sim_{G}$ is an equivalence relation on the projections $\Re$.

Proof. Suppose $E \sim_{G} F$. Then for each $t \in G$ there is $T_{t} \in \Re$ such that

$$
E=\sum_{t \in G} T_{t} T_{t}^{*}, \quad F=\sum_{t \in G} U_{t}^{*} T_{t}^{*} T_{t} U_{t}
$$

Then we have

$$
\begin{aligned}
\Phi(E) & =\sum \Phi\left(T_{t} T_{t}^{*}\right)=\sum \Phi\left(T_{t}\right) \Phi\left(T_{t}\right)^{*} \\
& =\sum\left(\Phi\left(T_{t}\right) \widetilde{U}_{t}\right)\left(\Phi\left(T_{t}\right) \widetilde{U}_{t}\right)^{*},
\end{aligned}
$$


and

$$
\begin{aligned}
\Phi(F) & =\sum \Phi\left(U_{t}^{*} T_{t}^{*} T_{t} U_{t}\right)=\sum \widetilde{U}_{t}^{*} \Phi\left(T_{t}^{*} T_{t}\right) \widetilde{U}_{t} \\
& =\sum\left(\Phi\left(T_{t}\right) \widetilde{U}_{t}\right)^{*}\left(\Phi\left(T_{t}\right) \widetilde{U}_{t}\right) .
\end{aligned}
$$

Thus by a result of Kadison and Pedersen [4, Thm. 4.1] $\Phi(E) \sim \Phi(F)$.

Conversely assume $\Phi(E) \sim \Phi(F)$. Then there is a partial isometry $V \in \mathfrak{B}$ such that $V V^{*}=\Phi(E), V^{*} V=\Phi(F)$. Say $V=\left(T_{s t^{-1}} U_{s t-1}\right)$. Then an easy calculation shows

$$
E=\sum_{t \in G} T_{t} T_{t}^{*}, \quad F=\sum_{t \in G} U_{t}^{*} T_{t}^{*} T_{t} U_{t},
$$

hence $E \sim{ }_{G} F$. The proof is complete.

Lemma 2. Let $S=\left(T_{s t-1} U_{s t-1}\right)$ belong to the center of $\mathfrak{B}$. Then for each $s \in G$ we have

(i) $T T_{s}=T_{s} U_{s} T U_{s}^{*}$ for all $T \in \Re$,

(ii) $T_{s y}=U_{y}^{*} T_{y s} U_{y}$ for all $y \in G$.

In particular $T_{e} \in \mathfrak{D}$. Furthermore, if $R \in \mathfrak{D}$ then $\Phi(R)$ belongs to the center of $\mathfrak{B}$.

Proof. Let $T \in \Re$. Then

$$
\left(T T_{s t^{-1}} U_{s t^{-1}}\right)=\Phi(T) S=S \Phi(T)=\left(T_{s t^{-1}} U_{s t^{-1}} T U_{t s}{ }^{-1} U_{s t^{-1}}\right),
$$

and (i) follows. Let $y \in G$. Then an easy computation shows

$$
\left(T_{s t^{-1} y^{-1}} U_{s t^{-1}}\right)=S \widetilde{U}_{y}=\widetilde{U}_{y} S=\left(U_{y} T_{y^{-1} s t^{-1}} U_{y}^{*} U_{s t^{-1}}\right) .
$$

Replacing $y$ by $y^{-1}$ and letting $t=e$, (ii) follows. By (i) $T_{e} T=T T_{e}$, so $T_{e} \in \mathfrak{C}$. By (ii) if $s=y^{-1}$ we find $T_{e}=U_{y}^{*} T_{e} U_{y}$, so $T_{e} \in \Re^{G}$, hence $T_{e} \in \mathfrak{D}$.

Finally let $R \in \mathfrak{D}$, and let $S^{\prime}=\left(S_{s t^{-1}} U_{s t^{-1}}\right) \in \mathfrak{B}$. Then we have

$$
\begin{aligned}
\Phi(R) S^{\prime} & =\left(R S_{s t^{-1}} U_{s t^{-1}}\right)=\left(S_{s t^{-1}} R U_{s t^{-1}}\right) \\
& =\left(S_{s t^{-1}} U_{s t^{-}} R\right)=S^{\prime} \Phi(R),
\end{aligned}
$$

hence $\Phi(R)$ belongs to the center of $\mathfrak{B}$. The proof is complete.

Lemma 3. Let $E$ be a projection in $\Re$. Let $D_{E}$ be the smallest operator in $\mathfrak{D}$ majorizing $E$. Then $D_{E}$ is a projection, and $\Phi\left(D_{E}\right)$ is the central carrier of $\Phi(E)$ in $\mathfrak{B}$.

Proof. Since $\mathfrak{D}$ is an abelian von Neumann algebra its positive operators form a complete lattice under infs and sups. Thus $D_{E}=$ g.l.b. $\{A \in \mathfrak{D}: E \leqq A \leqq I\}$, and $D_{E}$ is well defined. Since $E \leqq D_{E}$ and both operators commute we have $E=E^{2} \leqq D_{E}^{2}$. But $D_{E} \leqq I$, so $D_{E}^{2} \leqq$ 
$D_{E}$. Hence by minimality of $D_{E}, D_{E}=D_{E}^{2}$, so it is a projection. By Lemma $2 \Phi\left(D_{E}\right)$ is a central projection in $\mathfrak{B}$, hence if $C_{\Phi(E)}$ denotes the central carrier of $\Phi(E)$ in $\mathfrak{B}$, then $\Phi\left(D_{E}\right) \geqq C_{\Phi(E)}$. Now let $C_{\Phi(E)}=$ $\left(T_{s t^{-1}} U_{s t^{-1}}\right)$. By Lemma $2 T_{e} \in \mathfrak{D}$, and since $C_{\Phi(E)} \geqq \Phi(E), T_{e} \geqq E$. By definition of $D_{E}, T_{e} \geqq D_{E}$. But $\Phi\left(D_{E}\right) \geqq C_{\mathscr{Q}(E)}$, so $D_{E} \geqq T_{e}$, hence $T_{e}=$ $D_{E}$. The operator $\Phi\left(D_{E}\right)-C_{\Phi(E)}$ is positive and has zeros on the main diagonal. Therefore it is 0 , and $\Phi\left(D_{E}\right)=C_{\Phi(E)}$ as asserted.

LeMma 4. Let $E$ be a projection in $\Re$. Let $C_{E}$ be its central carrier in $\Re$, and let $D_{E}$ be as in Lemma 3. Then $D_{E}=D_{C_{E}}$.

Proof. Since $E \leqq C_{E}, D_{E} \leqq D_{C_{E}}$. But $D_{E} \in \mathbb{E}$ and $D_{E} \geqq E$, hence $D_{E} \geqq C_{E}$. Therefore by definition of $D_{C_{E}}, D_{E} \geqq D_{C_{E}}$, and they are equal.

LeMma 5. Let $E$ be a countably decomposable projection in $\Re$. Then $\Phi(E)$ is countably decomposable in $\mathfrak{B}$.

Proof. Let $x$ be a vector in ES. Then $x$ considered as a vector in $\sum_{t \in G} \oplus \mathfrak{S}_{t}$ belongs to $\mathfrak{S}_{e}$. Let $F$ be the support of $\omega_{x}$ in $E \Re E$. Then $F$ is countably decomposable, and $\omega_{x}$ is a faithful normal state of $F \Re F$. Let $\left\{F_{\alpha}\right\}_{\alpha \in J}$ be an orthogonal family of projections in $\mathfrak{B}$ such that $\sum_{\alpha \in J} F_{\alpha}=\Phi(F)$. Let $F_{\alpha}=\left(T_{s t-1}^{\alpha} U_{s t^{-1}}\right)$. Then $F_{\alpha} \leqq \Phi(F)$, so $T_{e}^{\alpha} \leqq F$, hence $T_{e}^{\alpha} \in F \Re F$. Furthermore, since $x \in \mathfrak{F}_{e}, \omega_{x}\left(F_{\alpha}\right)=\omega_{x}\left(T_{e}^{\alpha}\right)$. Thus we have

$$
1=\omega_{x}(F)=\omega_{x}(\Phi(F))=\sum \omega_{x}\left(F_{\alpha}\right)=\sum \omega_{x}\left(T_{e}^{\alpha}\right) .
$$

Therefore $\omega_{x}\left(T_{e}^{\alpha}\right)=0$ except for a countable number of $\alpha \in J$. But then $T_{e}^{\alpha}=0$ and hence $F_{\alpha}=0$ except for a countable number of $a \in$ $J$. Thus $\Phi(F)$ is countably decomposable in $\mathfrak{B}$. Now $E$ is a countable sum of orthogonal cyclic projections, hence $\Phi(E)$ is a countable sum of orthogonal countably decomposable projections. Hence $\Phi(E)$ is countably decomposable. The proof is complete.

Definition 2. We say a projection $E$ in $\Re$ is $\sim_{\sigma^{-}}$abelian if $E \Re E=$ $E D$.

Clearly a $\sim_{G^{-}}$abelian projection is abelian.

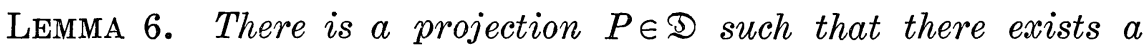
$\sim_{G}$-abelian projection $E \leqq P$ with $D_{E}=P$, and $I-P$ has no nonzero $\sim_{G}$-abelian subprojection.

Proof. Partially order the $\sim_{G}$-abelian projections in $\Re$ by $E \ll F$ if $E \leqq F$ and $D_{F-E} \leqq I-D_{E}$. Then in particular $D_{E} F=E$. Let $\left\{E_{\alpha}\right\}$ be a totally ordered set of $\sim_{G}$-abelian projections, and let $E=\sup E_{\alpha}$, 
so $E_{\alpha} \rightarrow E$ strongly. Then

$$
D_{E_{\alpha}} E=D_{E_{\alpha}} \lim _{\beta>\alpha} E_{\beta}=\lim _{\beta>\alpha} D_{E_{\alpha}} E_{\beta}=E_{\alpha},
$$

hence if $A \in \Re$ then

$$
E A E D_{E_{\alpha}}=E_{\alpha} A E_{\alpha}=A_{\alpha} E_{\alpha},
$$

where $A_{\alpha} \in \mathfrak{D} D_{E_{\alpha}}$. Now it is well known that if $Q_{\alpha}$ is an increasing net of projections, and $Q_{\alpha} \rightarrow Q$ strongly, then $C_{Q_{\alpha}} \rightarrow C_{Q}$ strongly. Thus

$$
\Phi\left(D_{E_{\alpha}}\right)=C_{\Phi\left(E_{\alpha}\right)} \rightarrow C_{\Phi(E)}=\Phi\left(D_{E}\right)
$$

by Lemma 3 , hence $D_{E_{\alpha}} \rightarrow D_{E}$ strongly. The same argument also shows

$$
D_{E-E_{\alpha}}=\lim _{\beta>\alpha} D_{E_{\beta}-E_{\alpha}} \leqq I-D_{E_{\alpha}} \text {. }
$$

Thus $E=E\left(I-D_{E_{\alpha}}\right)+E_{\alpha}$, and since $A_{\alpha}=A_{\alpha} D_{E_{\alpha}}$ we have $E A E D_{E_{\alpha}}=$ $A_{\alpha} E \in E \mathfrak{D}$. Since $D_{E_{\alpha}} \rightarrow D_{E}$ it follows that $E A E=\lim _{\alpha} E A E D_{E_{\alpha}} \in E \mathfrak{D}$. Therefore $E$ is $\sim_{G}$-abelian. Now let $E$ be a maximal $\sim_{G}$-abelian projection in $\Re$. Let $P=D_{E}$. Suppose $F$ is a $\sim_{G}$-abelian subprojection of $I-P$. Then $E+F$ is $\sim_{G}$-abelian. Indeed, if $A \in \Re$ then there are $A_{E} \in D_{E} \mathfrak{D}$ and $A_{F} \in D_{F} \mathfrak{D}$ such that

$$
\begin{aligned}
(E+F) A(E+F) & =E A E+F A F=E A_{E}+F A_{F} \\
& =(E+F)\left(A_{E}+A_{F}\right) \in(E+F) \mathfrak{D} .
\end{aligned}
$$

Thus $E+F$ is $\sim_{G}$-abelian. Since $E \ll E+F$, the maximality of $E$ implies $F=0$. The proof is complete.

Thus in order to prove Theorems 2 and 3 we may consider two cases separately, namely the case when $\Re$ has a $\sim_{G^{-}}$-abelian projection $E$ with $D_{E}=I$, and the case when $\Re$ has no nonzero $\sim_{G}$.abelian projection. We first treat the case with a $\sim_{G}$-abelian projection.

LEMma 7. Let $E$ be a $\sim_{G}$-abelian projection in $\Re$. Then $C_{E}$ is not G-equivalent to a proper central subprojection. Furthermore if $Q$ is a central projection such that $Q \leqq C_{E}$ then $Q=D_{Q} C_{E}$.

Proof. Let $Q$ be as in the statement of the lemma. Since $E$ is $\sim_{G}$-abelian there is an operator $D \in \mathscr{D}$ such that $Q E=D E$, hence, since $E \mathbb{C} \cong C_{E} \mathfrak{C}, Q=Q C_{E}=D C_{E}$, and $D \geqq Q$. By definition of $D_{Q}$, $D \geqq D_{Q}$. But $D_{Q} \geqq Q$, so $Q=Q C_{E} \leqq D_{Q} C_{E} \leqq D C_{E}=Q$, so that $Q=$ $D_{Q} C_{E}$. Now suppose $P$ is a projection in 5 such that $P \leqq C_{E}$ and $P \sim{ }_{G} C_{E}$. Then in particular by Lemma $1 \Phi(P) \sim \Phi\left(C_{E}\right)$, so they have the same central carrier in $\mathfrak{B}$, hence $D_{P}=D_{C_{E}}=D_{E}$ by Lemma 4 . By the preceding, $P=D_{P} C_{E}=C_{E}$. The proof is complete. 
Lemma 8. Let $E$ be $a \sim_{G}$-abelian projection in $\Re$. Let $Q$ be a central projection orthogonal to $C_{E}$. Then if $C_{E}$ and $C_{E}+Q$ are $G$ equivalent relative to $\mathfrak{E}$, i.e. the operators $T_{t}$ defining the equivalence belong to $\mathbb{E}$, then $Q=0$.

Proof. Let $P=C_{E}$ and assume $P \sim_{G} P+Q$ relative to $\sqrt{5 .}$ Then since $\mathbb{C}$ is abelian, for each $t \in G$ there is $A_{t} \in \mathbb{C}^{+}$such that $P=$ $\sum_{t \in G} A_{t}, P+Q=\sum_{t \in G} U_{t}^{*} A_{t} U_{t}$. Since $E \mathbb{C}=E \mathfrak{D}$ and $P \mathbb{C} \cong E \mathbb{C}$, we have $P \mathbb{C}=P \mathfrak{D}$. Since $A_{t} \leqq P$ there is $D_{t} \in \mathfrak{D}^{+}$such that $A_{t}=P D_{t}$. Thus we have

$$
\begin{aligned}
\sum P D_{t}=P & =P(P+Q)=\sum P U_{t}^{*} A_{t} U_{t} \\
& =\sum P U_{t}^{*} P D_{t} U_{t}=\sum P D_{t} U_{t}^{*} P U_{t}
\end{aligned}
$$

Now $P D_{t} U_{t}^{*} P U_{t} \leqq P D_{t}$ for all $t$, hence we have $P D_{t} U_{t}^{*} P U_{t}=P D_{t}$ for all $t$. I $\mathbf{e}^{t} E_{t}$ denote the range projection of $D_{t}$. Then $E_{t} \in \mathfrak{D}$. Since $U_{t}^{*} P U_{t} P D_{t}=P D_{t}, U_{t}^{*} P U_{t} P E_{t}=P E_{t}$. Thus $U_{t}^{*} P U_{t} \geqq P E_{t}$, and thus $U_{t}^{*} P E_{t} U_{t}=U_{t}^{*} P U_{t} E_{t} \geqq P E_{t}$. Consequently $P E_{t} \geqq U_{t} P E_{t} U_{t}^{*}$. By Lemma $7 P=C_{E}$ is $\sim_{G}$-finite relative to $\mathbb{E}$, hence so is $P E_{t}$. Therefore $P E_{t}=U_{t} P E_{t} U_{t}^{*}$, and $U_{t}^{*} P E_{t} U_{t}=P E_{t}$. Therefore we have

$$
U_{t}^{*} A_{t} U_{t}=U_{t}^{*} P D_{t} U_{t}=U_{t}^{*} P E_{t} U_{t} D_{t}=P E_{t} D_{t}=P D_{t}=A_{t},
$$

and $P=P+Q$, so that $Q=0$. The proof is complete.

LEMma 9. Suppose $E$ is $a \sim_{G^{-}}$abelian projection in $\Re$ with $D_{E}=$ I. Then $\Re$ is of type $I$, and there exists a faithful normal semifinite $G$-invariant trace on $\Re^{+}$.

Proof. Since $E$ is abelian $C_{E} \Re$ is of type $I$. Since every *-automorphism of $\Re$ preserves the type $I$ portion of $\Re$, and $D_{E}=I, \Re$ is of type $I$.

$E$ is a sum of orthogonal cyclic projections $E_{\alpha}$. If we can show the lemma for each $E_{\alpha}$ then it holds for $E$. Therefore we may assume $E$ is cyclic, say $E=\left[\Re^{\prime} x\right]$. Then $\omega_{x}$ is faithful on $E \Re E$, hence faithful on $E$ 〔 . If $A \geqq 0$ belongs to $C_{E} \Subset$ and $\omega_{x}(A)=0$, then $0=\omega_{x}(E A)$, so $E A=0$. Hence $A=A C_{E}=0$. Thus $\omega_{x}$ is faithful on $C_{E} \sqrt{5}$, so $C_{E}$ is a countably decomposable projection in 5 .

We shall now apply the previous theory to $\mathfrak{A}=\mathbb{E} \times G$ instead of $\mathfrak{B}=\mathfrak{R} \times G$. We use the same notation as before. By Lemma 7 $C_{E}$ is $\sim{ }_{G}$-finite. If $C_{E}=D_{E}=I$ then by Lemma $7 \quad[=D D$, and it is trivial that there exists a faithful normal semi-finite $G$-invariant trace on $\mathrm{C}^{+}$. Assume $C_{E} \neq I$. Then there is $s \in G$ such that $U_{s}^{*} C_{E} U_{s} \neq C_{E}$. Since by Lemma $7 C_{E}$ is $\sim_{G}$-finite, and $U_{s}^{*} C_{E} U_{s} \sim_{G} C_{E}, U_{s}^{*} C_{E} U_{s}$ is not a subprojection of $C_{E}$. Thus $Q=U_{s}^{*} C_{E} U_{s}\left(I-C_{E}\right) \neq 0$. Since $C_{E}$ is 
countably decomposable, so is $Q$, and hence $C_{E}+Q$. By Lemma 5 $\Phi\left(C_{E}+Q\right)$ is countably decomposable in $\mathfrak{X}$. Since $I=D_{E} \leqq D_{C_{E}}+Q$, the central carriers of $\Phi\left(C_{E}\right)$ and $\Phi\left(C_{E}+Q\right)$ are by Lemma 3 equal to $I$. If $\Phi\left(C_{E}\right)$ is properly infinite then by [1, Ch. III, $\S 8$, Cor. 5] $\Phi\left(C_{E}\right) \sim \Phi\left(C_{E}+Q\right)$, so by Lemma $1 C_{E} \sim_{G} C_{E}+Q$, contradicting Lemma 8. Thus $\Phi\left(C_{E}\right)$ is not properly infinite, and there is a nonzero central projection $P$ in $\mathfrak{A}$ such that $P \Phi\left(C_{E}\right)$ is nonzero and finite. Since the central carrier of $\Phi\left(C_{E}\right)$ is $I, P \mathscr{N}$ is semi-finite. Let $\varphi$ be a normal semi-finite trace on $\mathfrak{U}^{+}$with support $P$ such that $\varphi^{\prime}\left(\Phi\left(C_{E}\right)\right)<\infty$. For $A \in \mathbb{C}^{+}$define $\tau(A)=\varphi(\Phi(A))$. Then $\tau$ is a normal $G$-invariant trace because $\tau\left(U_{s}^{*} A U_{s}\right)=\varphi\left(\widetilde{U}_{s}^{*} \Phi(A) \widetilde{U}_{s}\right)=\varphi(\Phi(A))=\tau(A)$. Since $\tau\left(C_{E}\right)<\infty$ and $D_{C_{E}}=I$, $\tau$ is semi-finite, hence $\tau$ is a normal semi-finite $G$-invariant trace on $\mathfrak{C}^{+}$. Let $D$ be the support of $\tau$. Then $0 \neq D \in \mathfrak{D}$. Now apply the preceding to $(I-D) \subseteq$ and $E(I-D)$, and use Zorn's lemma to obtain a family $D_{\alpha}$ of orthogonal projections in $\mathscr{D}$ with sum $I$, and a normal semi-finite $G$-invariant trace $\tau_{\alpha}$ of $\mathbb{C}^{+}$with support $D_{\alpha}$. Let $\tau=\sum \tau_{\alpha}$. Then $\tau$ is a faithful normal semi-finite $G$-invariant trace on $\mathbb{C}^{+}$.

Now since $\Re$ is of type $I$ there is a faithful normal center valued trace $\psi$ on $\Re^{+}$such that $U_{s}^{*} \psi\left(U_{s} A U_{s}^{*}\right) U_{s}=\psi(A)$ for each $s \in G, A \in$ $\mathfrak{R}^{+}$, see [11, p. 3]. Then $\tau \circ \psi$ is a faithful normal semi-finite $G$ invariant trace on $\Re^{+}$, see [1, Ch. III, $\S 4$, Prop. 2]. The proof is complete.

LEMMA 10. Suppose $\Re$ is $\sim_{G}$-semi-finite and there are no nonzero $\sim_{G^{-}}$abelian projections in $\Re$. Then there is a faithful normal semi-finite $G$-invariant trace on $\mathfrak{R}^{+}$.

Proof. Let $E$ be a nonzero countably decomposable $\sim_{G}$-finite projection in $\Re$. Since $E$ is not $\sim_{G}$-abelian there is a projection $H \in$ $E \Re E$ such that $H \neq E D_{H}$. Let $F=H+\left(I-D_{H}\right) E$. Then $F \leqq E$, $F \neq E$, and $D_{F}=D_{H}+\left(I-D_{H}\right) D_{E}=D_{E} \cdot \Phi(F)$ is not properly infinite in $\mathfrak{B}$. Indeed, if it were, then since $\Phi(E)$ is countably decomposable by Lemma 5, [1, Ch. III, §8, Cor. 5] would imply $\Phi(F) \sim \Phi(E)$, hence by Lemma $1, F \sim_{G} E$, contradicting the $\sim_{G}$-finiteness of $E$. Therefore there is a nonzero central projection $P$ in $\mathfrak{B}$ such that $P \Phi(F)$ is finite and nonzero. Thus $P \Phi\left(D_{E}\right) \mathfrak{B}=P \Phi\left(D_{F^{\prime}}\right) \mathfrak{B}$ is semi-finite and nonzero. Let $\varphi$ be a normal semi-finite trace on $\mathfrak{B}$ with support $P \Phi\left(D_{E}\right)$ such that $\varphi(\Phi(F))<\infty$. For $A \in \Re^{+}$define $\tau(A)=\varphi(\Phi(A))$. As in the proof of Lemma $9 \tau$ is a normal $G$-invariant trace on $\mathfrak{R}^{+}$. Since $\tau(F)<\infty$ there is a nonzero central projection $Q$ in $\Re$ such that $\tau$ is faithful and semi-finite on $Q \Re$ [1, Ch. I, $\S 6$, Cor. 2]. Since $\tau$ is $G$-invariant $Q \in \mathfrak{D}$. Now a Zorn's Lemma argument completes the proof just as in Lemma 9. 
Proof of Theorem 2. By Lemma 6 there is a projection $P \in \mathfrak{D}$ such that there exists a $\sim_{G}$-abelian projection $E \in P \Re$ with $D_{E}=P$, and $I-P$ has no nonzero $\sim_{G}$-abelian subprojection. By Lemma 9 there is a faithful normal semi-finite $G$-invariant trace $\tau_{1}$ on $P \Re^{+}$. If $\Re$ is $\sim_{G}$-semi-finite then by Lemma 10 there is a faithful normal semi-finite $G$-invariant trace $\tau_{2}$ on $(I-P) \Re^{+}$. Thus $\tau=\tau_{1}+\tau_{2}$ is a faithful normal semi-finite $G$-invariant trace on $\Re^{+}$.

Conversely assume there exists a faithful normal semi-finite $G$ invariant trace $\tau$ on $\Re^{+}$. Suppose $E$ is a projection in $\Re$ such that $\tau(E)<\infty$. Since $E \sim{ }_{G} F$ implies $\tau(E)=\tau(F)$ it is clear that $E$ is $\sim_{G}$-finite. Thus $\Re$ is $\sim_{G}$-semi-finite. The proof is complete.

LEMMA 11. Suppose $\mathbb{C}$ is countably decomposable and $\Re$ is $\sim_{G^{-}}$ finite. Then there is a faithful normal finite G-invariant trace on $\Re$.

Proof. Since $\Re$ is $\sim_{G}$-finite $\mathfrak{R}$ is in particular finite. By [1, Ch. III, $\S 4$, Thm. 3] there is a unique center valued trace $\psi$ on $\Re$ which is the identity on $\widetilde{c}$. By uniqueness $\psi$ is $G$-invariant, so if $\tau$ is a faithful normal finite $G$-invariant trace on $\mathbb{E}$, then $\tau \circ \psi$ is one on $\Re$. Therefore we may assume $\Re=\mathfrak{c}$. Now there exists a projection $P \in \mathfrak{D}$ such that $P \mathbb{C}=P \mathfrak{D}$, and $G$ is freely acting on $(1-P) \mathfrak{C}$, i.e. for each projection $E \neq 0$ in $(I-P)$ ( there is a nonzero subprojection $F$ of $E$ and $s \in G$ such that $U_{s}^{*} F U_{s} \leqq I-F$, see e.g. [5]. Since $I$ is countably decomposable, so is $P$, and there is a faithful normal state on $P \mathbb{C}$, hence a faithful normal finite $G$-invariant trace on $P$ C . We may thus assume $G$ is freely acting. Let $F$ be a nonzero projection in $\sqrt{5}$ and $s$ an element in $G$ such that $U_{s}^{*} F U_{s} \leqq I-F$. Let $E=I-F$. Then $D_{E}=I$, and $F \prec_{G} E . \quad$ As in the proof of Lemma $10 \Phi(E)$ is not properly infinite, so we can choose a central projection $P \neq 0$ in $\mathfrak{B}$ such that $P \Phi(E)$ is finite. Since $F \prec_{G} E, \Phi(F) \prec \Phi(E)$, by Lemma 1 , hence $P \Phi(F) \prec P \Phi(E)$, so $P \Phi(F)$ is finite. Thus $P=$ $P \Phi(E)+P \Phi(F)$ is finite in $\mathfrak{B}$, and $P \mathfrak{B}$ is finite. Since $I$ is countably decomposable in $\mathfrak{C}(=\Re) \Phi(I)$ is countably decomposable in $\mathfrak{B}$ by Lemma 5 , hence so is $P$. Therefore by [1, Ch. I, $\S 6$, Prop. 9] there is a faithful normal finite trace $\varphi$ on $P \mathfrak{B}$. Then $\tau$ defined by $\tau(A)=$ $\phi(\Phi(A))$ is a normal finite $G$-invariant trace on 5 with support $D \neq 0$ in D. A Zorn's Lemma argument now gives a family $\tau_{\alpha}$ of normal finite $G$-invariant traces on $\sqrt{5}$ with orthogonal supports $D_{\alpha}$ in D. Since $I$ is countably decomposable the family $\left\{\tau_{\alpha}\right\}$ is countable, and by multiplying each $\tau_{\alpha}$ by a convenient positive scalar we may assume $\sum \tau_{\alpha}\left(D_{\alpha}\right)=1$. Thus if $\tau=\sum \tau_{\alpha}$, then $\tau$ is a faithful normal finite $G$-invariant trace on $\sqrt{ }$. The proof is complete. 
Proof of Theorem 3. Suppose there is a faithful normal finite $G$-invariant trace $\tau$ on $\Re$. Then $I$ is $\sim_{G}$-finite, for if $E$ is a projection in $\Re$ which is $G$-equivalent to $I$ then $\tau(E)=\tau(I)$, hence $\tau(I-E)=0$, hence $I-E=0$, since $\tau$ is faithful. Thus $\Re$ is $\sim_{G}$-finite. Again since $\tau$ is faithful, its support $I$ is countably decomposable, i.e. $R$ is countably decomposable. The converse follows from Lemma 11.

CoRollary. If $\Re$ is $\sim_{G^{-}}$-semi-finite then $\mathfrak{B}$ is semi-finite. If $\Re$ is $\sim_{G}$-finite and there is an orthogonal family of countably decomposable projections in $\mathfrak{D}$ with sum $I$, then $\mathfrak{B}$ is finite.

Proof. If $\Re$ is $\sim_{G}$-semi-finite, then by Theorem 2 there is a faithful normal semi-finite $G$-invariant trace on $\Re$. Thus there is a faithful normal semi-finite trace on $\mathfrak{B}$ by [1, Ch. I, §9, Prop. 1], hence $\mathfrak{B}$ is semi-finite. If $P$ is a projection in $\mathfrak{D}$ then by Lemma 2 $\Phi(P)$ is a central projection in $\mathfrak{B}$. Thus in order to show the last part of the corollary we may assume $I$ is countably decomposable. Then by Theorem 3 there is a faithful normal finite $G$-invariant trace on $\Re$, hence by $[1, \mathrm{Ch} . \mathrm{I}, \S 9$, Prop. 1] there is a normal finite trace on $\mathfrak{B}$, so $\mathfrak{B}$ is finite. The proof is complete.

REMarK 5. G. K. Pedersen has pointed out that the corollary can be sharpened. Indeed one can show that if $E$ is a projection in $\Re$ then $E$ is $\sim_{G}$-finite if and only if $\Phi(E)$ is finite in $\mathfrak{B}$. In particular $\Re$ is $\sim_{G}$-finite if and only if $\mathfrak{B}$ is finite.

4. G-finite von Neumann algebras. Let notation be as in Theorem 1. Following [7] we say $\Re$ is $G$-finite if there is a family $\mathscr{F}$ of normal $G$-invariant states which separate $\mathfrak{R}^{+}$, i.e. if $A \in \mathfrak{R}^{+}$, and $\omega(A)=0$ for all $\omega \in \mathscr{F}$, then $A=0$. For semi-finite von Neumann algebras it would be natural to compare this concept with those of $\sim_{G}$-finite and $\sim_{G^{-}}$-semi-finite. Since a $\sim_{G}$-finite von Neumann algebra is necessarily finite we cannot expect a $G$-finite semi-finite von Neumann algebra to be $\sim_{G}$-finite. We say $G$ acts ergodically on (5) if $\mathfrak{D}\left(=\mathbb{E} \cap \mathfrak{R}^{G}\right)$ is the scalars.

THEOREM 4. Let $\Re$ be a semi-finite von Neumann algebra acting on a Hilbert space $\mathfrak{K}_{\text {. }}$ Let $G$ be a group and $t \rightarrow U_{t}$ a unitary representation of $G$ on $\mathfrak{S}$ such that $U_{t}^{*} \Re U_{t}=\mathfrak{R}$ for all $t \in G$. Assume either that $G$ acts ergodically on the center of $\Re$ or the center is elementwise fixed under $G$. Then $\Re$ is $G$-finite if and only if there is a faithful normal semi-finite $G$-invariant trace $\tau$ on $\mathfrak{R}^{+}$and an orthogonal family $\left\{E_{\alpha}\right\}$ of $G$-invariant projections in $\mathfrak{R}$ with sum $I$ and $\tau\left(E_{\alpha}\right)<\infty$ for each $\alpha$. 
Proof. Assume $\Re$ is $G$-finite. Suppose first that $G$ acts ergodically on the center $\mathfrak{C}$ of $\Re$, and suppose $\omega$ is a faithful normal $G$-invariant state on $\Re$. Then by [11] there is a faithful normal semifinite $G$-invariant trace on $\Re^{+}$, hence by Theorem $2 \Re$ is $\sim_{G^{-}}$-semi-finite. In general, by Zorn's Lemma there is a family $\left\{\omega_{\alpha}\right\}$ of normal Ginvariant states with orthogonal supports $E_{\alpha}$ such that $\sum E_{\alpha}=I$. Then each $E_{\alpha}$ is $G$-invariant, and by the first part of the proof $E_{\alpha} \Re E_{\alpha}$ is $\sim_{G}$-semi-finite. In particular, $E_{\alpha}$ is the sup of an increasing net of $\sim_{G}$-finite projections. Let $F$ be a projection in $\Re$. We show $F$ has a nonzero $\sim_{G}$-finite subprojection. By the above considerations there are $E_{\alpha}$ and a $\sim_{G}$-finite subprojection $F_{\alpha}$ of $E_{\alpha}$ such that $C_{F_{\alpha}} F \neq$ 0 . Let $F_{1}=C_{F_{\alpha}} F$. Then there is a nonzero subprojection $F_{0}$ of $F_{1}$ such that $F_{0} \lesssim F_{\alpha}$. Say $F_{0} \sim G_{\alpha} \leqq F_{\alpha}$. Since $F_{\alpha}$ is $\sim_{G}$-finite, so is $G_{\alpha}$. Indeed, if $G_{\alpha} \sim{ }_{G} H \leqq G_{\alpha}$ then by Lemma $1 \Phi\left(G_{\alpha}\right) \sim \Phi(H)$, hence $\Phi\left(F_{\alpha}\right)=\Phi\left(G_{\alpha}\right)+\Phi\left(F_{\alpha}-G_{\alpha}\right) \sim \Phi(H)+\Phi\left(F_{\alpha}-G_{\alpha}\right)$, so again by Lemma 1, $F_{\alpha} \sim_{G} H+F_{\alpha}-G_{\alpha}$, so that $H=G_{\alpha}$ by finiteness of $F_{\alpha}$. Thus $G_{\alpha}$ is $\sim_{G}$-finite. Since $G_{\alpha}$ is in particular finite there is by [1, Ch. III, §2, Prop. 6] a unitary operator $U \in \Re$ such that $U F_{0} U^{-1}=G_{\alpha}$. But then $F_{0}$ is $\sim_{G}$-finite, for if $F_{0} \sim_{G} F_{2} \leqq F_{0}$ then $U F_{2} U^{-1} \sim F_{2} \sim_{G} G_{\alpha}$, so by transitivity $U F_{2} U^{-1} \sim_{G} G_{\alpha}$. Since $U F_{2} U^{-1} \leqq G_{\alpha}$, they are equal by finiteness of $G_{\alpha}$, so $F_{2}=F_{0}$, and $F_{0}$ is $\sim_{G}$-finite. Therefore the projection $F$ has a nonzero $\sim_{G}$-finite subprojection $F_{0}$, and $\Re$ is $\sim_{G}$-semifinite.

Next assume $\mathbb{E}=\mathfrak{D}$. Then every normal semi-finite trace on $\mathfrak{R}^{+}$ is $G$-invariant [10, Cor. 2.2], so there exists a faithful normal semifinite $G$-invariant trace on $\Re^{+}$, hence by Theorem $2, \Re$ is $\sim_{G^{-}}$-semi-finite.

Let by Theorem $2 \tau$ be a faithful normal semi-finite $G$-invariant trace on $\Re^{+}$. Let $\left\{\omega_{\alpha}\right\}$ be as before with orthogonal supports $\left\{E_{\alpha}\right\}$. Then there is a positive self-adjoint operator $H_{\alpha} \in L^{1}(\Re, \tau)$ affiliated with $\Re^{G}$ such that $\omega_{\alpha}(T)=\tau\left(H_{\alpha} T\right)$ for $T \in \Re$, see e.g. [1, Ch. I, $\S 6$, no. 10]. Let $E$ be a spectral projection of $H_{\alpha}$ with $\tau(E)<\infty$. Then $E$ is $G$-invariant. A Zorn's Lemma argument now gives an orthogonal family of $G$-invariant projections in $\Re$ with sum $I$ and finite trace.

Conversely assume $\mathfrak{R}$ has a faithful normal semi-finite $G$-invariant trace $\tau$ and an orthogonal family $\left\{E_{\alpha}\right\}$ of nonzero $G$-invariant projections with sum $I$ such that $\tau\left(E_{\alpha}\right)<\infty$. Let $c_{\alpha}=\tau\left(E_{\alpha}\right)^{-1}$, and let $\omega_{\alpha}(T)=c_{\alpha} \tau\left(E_{\alpha} T\right)$. Then $\left\{\omega_{\alpha}\right\}$ is a separating family of normal $G$ invariant states on $\Re$, hence $\Re$ is $G$-finite. The proof is complete.

The above theorem is probably true without the assumptions of the action of $G$ on $\mathbb{C}$. A direct proof of this would be quite interesting.

5. Abelian von Neumann algebras. Assume $\mathfrak{R}$ is an abelian von Neumann algebra acting on a Hilbert space $\mathfrak{F}$. Let $G$ be a group and suppose $t \rightarrow U_{t}$ is a unitary representation of $G$ on $\mathfrak{S}$ such that 
$U_{t}^{*} \Re U_{t}=\Re$ for all $t \in G$. We say two projections $E$ and $F$ in $\Re$ are equivalent in the sense of Hopf and write $E \sim_{H} F$ if there are an orthogonal family of projections $\left\{E_{\alpha}\right\}_{\alpha \in J}$ in $\Re$ and $t_{\alpha} \in G$, for $\alpha \in J$, such that $E=\sum E_{\alpha}, F=\sum U_{t_{\alpha}}^{*} E_{\alpha} U_{t_{\alpha}}$. Since each $U_{t_{\alpha}}^{*} E_{\alpha} U_{t_{\alpha}}$ is a projection, and their sum is a projection, they are all mutually orthogonal. Since we can collect the $E_{a}$ 's for which $t_{\alpha}$ coincide the definition of equivalence in the sense of Hopf is equivalent to the existence of an orthogonal family of projections $\left\{E_{t}\right\}_{t \in G}$ in $\Re$ such that $E=\sum_{t \in G} E_{t}, F=\sum_{t \in G} U_{t}^{*} E_{t} U_{t}$. This equivalence was introduced by Hopf [3]. Just as for $\sim_{G}$ we define $\sim_{H}$-finite, $\sim_{H}$-semi-finite, and $\prec_{H}$. Note that if $E \sim_{H} F$ as above, if we let $T_{t}=E_{t}$, then $E=\sum T_{t} T_{t}^{*}, F=\sum U_{t}^{*} T_{t}^{*} T_{t} U_{t}$, so $E \sim_{G} F$. If we assume $\Re$ is countably decomposable, we shall now prove the converse via a proof which makes use of the known results on invariant measures if $\Re$ is $\sim_{H}$-finite and $\sim_{H^{-}}$-semi-finite. A direct proof would be more desirable.

Theorem 5. Assume $\mathfrak{R}$ is countably decomposable, and let notation be as above. Then two projections $E$ and $F$ in $\Re$ are G-equivalent if and only if they are equivalent in the sense of Hopf.

Outline of proof. It remains to be shown that if $E \sim_{G} F$ then $E \sim_{H} F$. Assume $E \sim_{G} F$. By Lemma $1 \Phi(E) \sim \Phi(F)$, so they have the same central carrier $C$. By Lemma $3 \Phi\left(D_{E}\right)=C=\Phi\left(D_{F}\right)$, so $D_{E}=D_{F}$. Suppose first $E$ and $F$ are such that $E P$ and $F P$ are $\sim_{H}$-infinite for all nonzero projections $P \in \mathfrak{D}$. In a von Neumann algebra two properly infinite countably decomposable projections with the same central carries are equivalent [1, Ch. III, §8, Cor. 5]. Using the comparison theory for $\Re$ with the Hopf ordering $\prec_{H}$, as developed in [6], see also [9], we can modify the proof of the quoted result for von Neumann algebras, to show $E \sim_{H} F$. If $E$ is $\sim_{H}$-finite then since $D_{E}=D_{F}$, we may assume $\Re$ is $\sim_{H}$-semi-finite, so by [6] there is a faithful normal semi-finite $G$-invariant trace $\tau$ on $\mathfrak{R}^{+}$such that $\tau(E)<\infty$. From the comparison theorem on $\Re[6$, Lem. 16], or [9, Lem. 2.7], there exist two orthogonal projections $P$ and $Q$ in D with sum $I$ such that $P E \prec_{H} P F$ and $Q F \prec_{H} Q E$. Since $P E \sim_{G} P F$ we have $\tau(P E)=\tau(P F)$. But if a proper subprojection $F_{0}$ of $P F$ is such that $P E \sim_{H} F_{0}$ then $\tau(P E)=\tau\left(F_{0}\right)<\tau(P F)=\tau(P E)$, a contradiction. Thus $P E \sim_{H} P F$, and similary $Q E \sim_{H} Q F$. Thus $E \sim_{H} F$, and the proof is complete.

REMARK 6 . Theorem 5 is undoubtably true without the assumption that $\Re$ is countably decomposable. If $E$ is $\sim_{H}$-finite then it is still possible to find $\tau$ as above. If $E$ is $\sim_{H}$-infinite the above 
proof works as long as $E$ is countably decomposable. Otherwise the theorem seems to be more difficult to prove, cf. proof of [4, Thm. 4.1].

\section{REFERENCES}

1. J. Dixmier, Les algèbres d'opérateurs dans l'espace hilbertien, Gauthier-Villars, Paris 1957.

2. P. R. Halmos, Invariant measures, Ann. Math., 48 (1947), 735-754.

3. E. Hopf, Theory of measures and invariant integrals, Trans, Amer. Math. Soc., 34 (1932), 373-393.

4. R. V. Kadison and G. K. Pedersen, Equivalence in operator algebras, Math. Scand., 27 (1970), 205-222.

5. R. R. Kallman, A generalization of free action, Duke Math., J., 36 (1969), 781-789.

6. Y. Kawada, Über die Existenz der invarianten Integrale, Japan J. Math., 19 (1944), 81-95.

7. I. Kovács and J. Szücs, Ergodic type theorems in von Neumann algebras, Acta Sci. Math., 27 (1966), 233-246.

8. F. J. Murray and J. von Neumann, On rings of operators, Ann. Math., 37 (1937), 116-229.

9. E. St $\phi$ rmer, Large groups of automorphisms of $C^{*}$-algebras, Commun. Math. Phys., 5 (1967), 1-22.

10. - States and invariant maps of operator algebras, J. Fnal. Anal., 5 (1970), 44-65.

11. - Automorphisms and invariant states of operator algebras, Acta Math., 127 (1971), 1-9.

Received September 27, 1971.

UNIVERSITY OF OSLO 



\section{PACIFIC JOURNAL OF MATHEMATICS}

\section{EDITORS}

H. SAMELSON

Stanford University

Stanford, California 94305

C. R. Hовву

University of Washington

Seattle, Washington 98105
J. DugundJI

Department of Mathematics University of Southern California

Los Angeles, California 90007

RICHARD ARENS

University of California

Los Angeles, California 90024

\section{ASSOCIATE EDITORS}

E. F. BECKENBACH

B. H. NeumanN

F. WOLF

K. YoSHIDA

\section{SUPPORTING INSTITUTIONS}

UNIVERSITY OF BRITISH COLUMBIA

CALIFORNIA INSTITUTE OF TECHNOLOGY

UNIVERSITY OF CALIFORNIA

MONTANA STATE UNIVERSITY

UNIVERSITY OF NEVADA

NEW MEXICO STATE UNIVERSITY

OREGON STATE UNIVERSITY

UNIVERSITY OF OREGON

OSAKA UNIVERSITY
UNIVERSITY OF SOUTHERN CALIFORNIA

STANFORD UNIVERSITY

UNIVERSITY OF TOKYO

UNIVERSITY OF UTAH

WASHINGTON STATE UNIVERSITY

UNIVERSITY OF WASHINGTON

AMERICAN MATHEMATICAL SOCIETY

NAVAL WEAPONS CENTER

Printed in Japan by International Academic Printing Co., Ltd., Tokyo, Japan 


\section{Pacific Journal of Mathematics}

\section{Vol. 44, No. $1 \quad$ May, 1973}

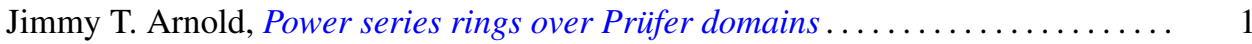

Maynard G. Arsove, On the behavior of Pincherle basis functions . . . . . . . . . 13

Jan William Auer, Fiber integration in smooth bundles ................. 33

George Bachman, Edward Beckenstein and Lawrence Narici, Function algebras

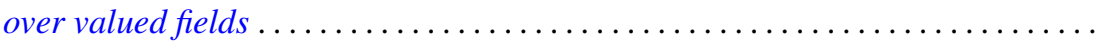

Gerald A. Beer, The index of convexity and the visibility function . . . . . . . . . . .

James Robert Boone, A note on mesocompact and sequentially mesocompact

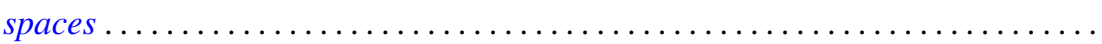

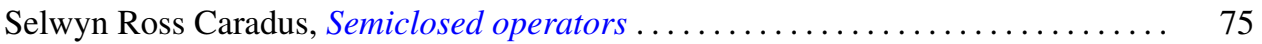

John H. E. Cohn, Two primary factor inequalities . . . . . . . . . . . . . . . 81

Mani Gagrat and Somashekhar Amrith Naimpally, Proximity approach to

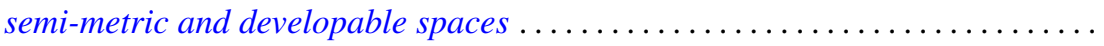

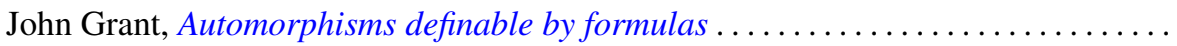

Walter Kurt Hayman, Differential inequalities and local valency ..............

Wolfgang H. Heil, Testing 3-manifolds for projective planes . . . . . . . . . . . . .

107

Melvin Hochster and Louis Jackson Ratliff, Jr., Five theorems on Macaulay

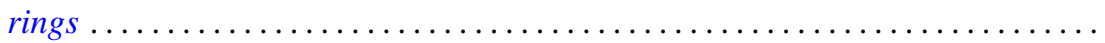

Thomas Benton Hoover, Operator algebras with reducing invariant subspaces ....

James Edgar Keesling, Topological groups whose underlying spaces are separable

Fréchet manifolds...

Frank Leroy Knowles, Idempotents in the boundary of a Lie group . .

191

George Edward Lang, The evaluation map and EHP sequences ...

201

Everette Lee May, Jr, Localizing the spectrum . . . . . . . . . . . .

211

Frank Belsley Miles, Existence of special $K$-sets in certain locally compact abelian groups.

Susan Montgomery, A generalization of a theorem of Jacobson. II . .

T. S. Motzkin and J. L. Walsh, Equilibrium of inverse-distance forces in

three-dimensions.

Arunava Mukherjea and Nicolas A. Tserpes, Invariant measures and the converse

of Haar's theorem on semitopological semigroups .

James Waring Noonan, On close-to-convex functions of order $\beta$

Donald Steven Passman, The Jacobian of a growth transformation

Dean Blackburn Priest, A mean Stieltjes type integral ........ .

Joe Bill Rhodes, Decomposition of semilattices with applications to topological

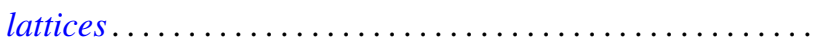

Claus M. Ringel, Socle conditions for $\mathrm{QF}-1$ rings ..........

Richard Rochberg, Linear maps of the disk algebra

Roy W. Ryden, Groups of arithmetic functions under Dirichlet convolution . .

Michael J. Sharpe, A class of operators on excessive functions

Erling Stormer, Automorphisms and equivalence in von Neumann algebras ..

Philip C. Tonne, Matrix representations for linear transformations on series

analytic in the unit disc. 